\title{
The Impact of Throughput Fluctuation on Quality of Experience for YouTube Service
}

\author{
Arisa Vilaikruad ${ }^{1}$, Monthippa Uthansakul ${ }^{1}$, Peerapong Uthansakul ${ }^{1+}$ and Settawit Poochaya ${ }^{1}$ \\ School of Telecommunication Engineering, Suranaree University of Technology, NakhonRatchasima,
} Thailand 30000

\begin{abstract}
With the massive demand of YouTube service in $4 \mathrm{G}$ mobile network, it is the urgent and important issue for service providers to maintain the satisfaction of all users. Although the throughput in $4 \mathrm{G}$ network is typically high but the user cannot avoid experiencing the fluctuation of data downloading. In general, this fluctuation cannot be predicted due to real-time user environments. For video streaming service like YouTube, it is expected to have the least fluctuation. Hence, it is interesting to see the impact of the throughput fluctuation on the satisfaction of users in order to improve the network quality. This paper presents the novel conclusion on the relationship between throughput fluctuation and the quality of experience in term of user satisfaction for YouTube service.
\end{abstract}

Keywords: QoE, QOS, MOS, Throughput Fluctuations.

\section{Introduction}

Currently, the increasing demand for mobile phones has been exponentially growing since people trend to reply on multimedia videos nowadays. The report by Cisco [1] estimated that the global mobile video traffic will increase 13-fold from 2014 to 2019 accounted for $72 \%$ of data traffic on mobile devices. As a result, network providers lately focus on improving network quality in order to provide a higher quality video to fulfil the satisfaction of customers. So far, most of network providers traditionally focus on only Quality of Service (QoS) and signal strength to improve the network efficiency. However, some mobile users cannot access the service. This is because a number of users need to access the service at the same time. For this case, the network providers cannot meet the user satisfaction. Alternatively, Quality of Experience (QoE) is an interesting parameter to indicate the real user satisfaction. As long as the network providers know the QoE from users, they will be able to provide the services according to the user demand.

Therefore, this paper proposes a focus on understanding the relationship between user satisfactions on YouTube service with the video quality. In this paper, the quality indicator is a Mean Opinion Score (MOS) which reflects many parameters indicating the service quality such as packet Loss, delay, Jitter, bandwidth, latency and network throughput [2-3]. Please note that a downlink throughput is chosen for this study as it is widely used and well recognized for the network efficiency monitoring. From literatures, some researchers have focused on Key Performance Indicator (KPI) to create the downlink throughput fluctuation model from bandwidth fluctuations to analyse the QoE on the traffic issue of mobile network [4-5]. However, all of them have been demonstrated only in laboratory. Therefore, this paper further investigates the fluctuation effect on the real users in real circumstances of $4 \mathrm{G}$ mobile network.

The remainder of the paper is as follows. In Section 2, the background overviews of research related to the system quality indicators such as QoS and QoE is presented. Section 3 discusses on the details of

\footnotetext{
+ Corresponding author. Tel.: +66850865588; fax: +6644224603.

E-mail address: uthansakul@ sut.ac.th
} 
experiments to achieve QoE in real circumstances. Then, the experimental results and discussions will be presented in Section 4. Finally, Section 5 concludes the paper.

\section{Background Overviews}

The definition of QoS has been addressed by ITU in 1988 as the effect of service performance which determines the level of user satisfaction for mobile networks [6] in order to allocate the available resources for the most efficient networks. The definition of QoE has been addressed by ITU-T in 2008 as the acceptance of the applications or services which is perceived subjectively by the end-user [7]. The brief details of these 2 parameters are as follows.

\subsection{QoE Evaluations}

The evaluation of QoE is divided into 2 methods. The first one is called as a subject evaluation method which is the quality of the opinion referring to a feeling or satisfaction of users. The second method is an objective evaluation method. It is a relationship of mathematical equations that have been proposed by ITUT P.862 [8]. Please note that this paper focuses on the subject evaluation method because the mobile networks have the specific environments beyond the formula of ITU-T P.862.

\subsection{QoS Parameters}

The downlink throughput is an important factor to determine the user satisfaction when users are watching the video on YouTube service. This is because throughput or data transmission speed creates a buffer which directly delays the video downloading. The users can directly experience the effect of delaying while watching YouTube video.

Fig. 1 shows the downlink throughput when watching YouTube videos during 1 minute. This data was originally recorded using the application named Azenqos on mobile phones. As we can see in the figure, the downlink throughput changes every second. This change is called as throughput fluctuations. In this example, the average throughput is $2.28 \mathrm{Mbps}$ while the throughput in each second is varied from $0-16 \mathrm{Mbps}$.

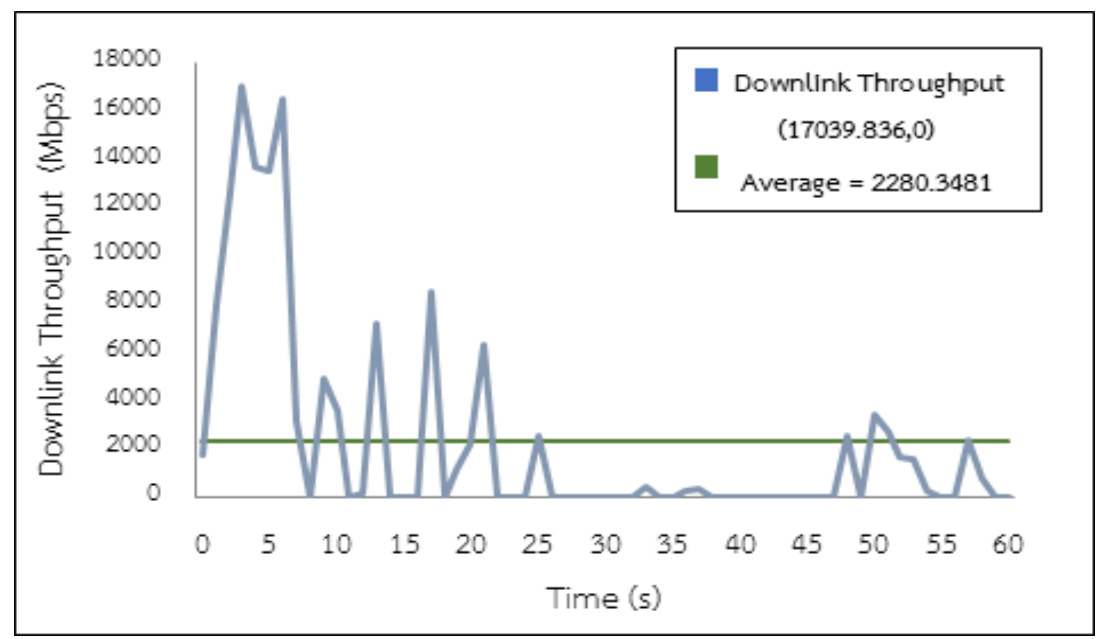

Fig. 1: Downlink throughput fluctuation is measured when watching YouTube videos within 1 minute.

In order to measure the degree of fluctuations, the Standard Deviation (SD) of downlink throughput can be the representative of fluctuation variation. The measured throughputs are collected from Azenqos application which is a popular tool for $4 \mathrm{G}$ mobile networks. Then the correlation analysis using SD of throughputs forms the relationship between Mean Opinion Score (MOS) and SD. Fig. 2 shows the diagram of subjective evaluation method to find the relationship between fluctuations and QOE on $4 \mathrm{G}$ mobile networks. 

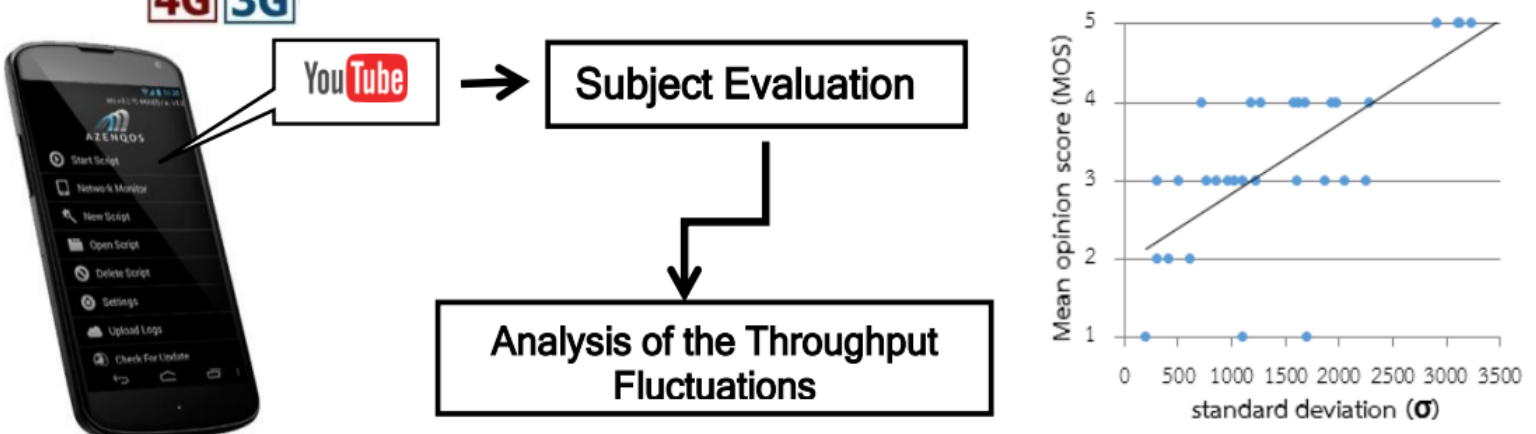

AzenopsApplication

Fig. 2: Subjective evaluation method to find the relationship between fluctuations and QoE.

\section{Experimental Results and Discussions}

\subsection{Subject Evaluation Method}

The subject evaluation method aims to measure the quality of video [9] based on the viewpoint of users through their questionnaires. This work surveyed 90 participants who watch YouTube video for 1 minute. The 40 participants were female and the others were male, whose ages were ranged from 21 to 24 years old. All participants watched the same YouTube video on the mobile phone at 1080 p quality. After watching video, users commented on the video whether they were satisfied with the quality of video or not. They can mark a measure of satisfaction with the quality of video over five levels from 1 to 5, also known as the Mean Opinion Score (MOS) [10]. Table 1 shows the meaning of MOS levels and their quality.

Table1: MOS versus video quality.

\begin{tabular}{|l|l|l|}
\hline MOS & QUALITY & \multicolumn{1}{c|}{ IMPAIRMENT } \\
\hline 5 & Excellent & Imperceptible \\
\hline 4 & Good & Perceptible but not annoying \\
\hline 3 & Fair & Slightly annoying \\
\hline 2 & Poor & Annoying \\
\hline 1 & Bad & Very annoying \\
\hline
\end{tabular}

\subsection{Throughput Fluctuations}

The measured locations are randomly spread throughout the area of Suranaree University of Technology, Muang District, Nakhon Ratchasima, Thailand. Fig. 3 shows the map of evaluation area which has an approximate population of 17,000 people (exploration data Friday, August 19, 2016 at 3:47 pm.).

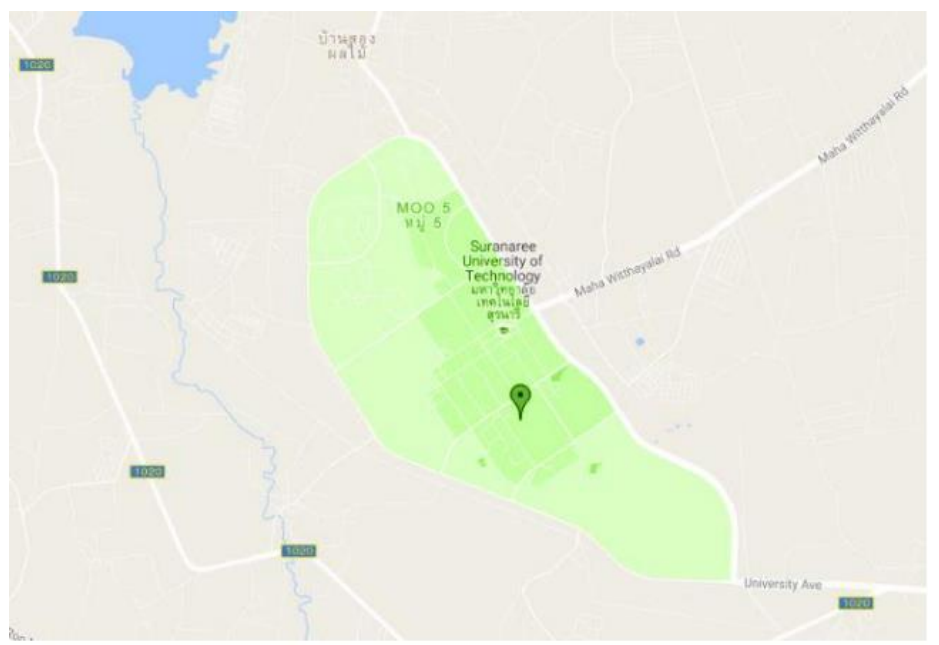

Fig. 3: the map of measured are. 
Fig. 4 shows the relationship between MOS and standard deviation $(\sigma)$ by subjective evaluation method for YouTube service with video quality of $1080 \mathrm{p}$, when the average throughput is (a) 2100-2500 kbps (b) 1900-2100 kbps (c) 1200-1400 kbps (d) 900-1200 kbps and (e) 500-900 kbps. In Fig. 4(a), it is clearly seen that all users are very satisfied with the YouTube service no matter what the standard deviation is high or low. This is because the downlink throughput is too large to be impaired by fluctuation. Also the YouTube service has some buffers that are tolerance to this fluctuation. This buffer can be worked only when the download speed is fast enough. Otherwise, the buffers cannot help to ignore the fluctuation as seen in Fig. 4 (b) to (e).

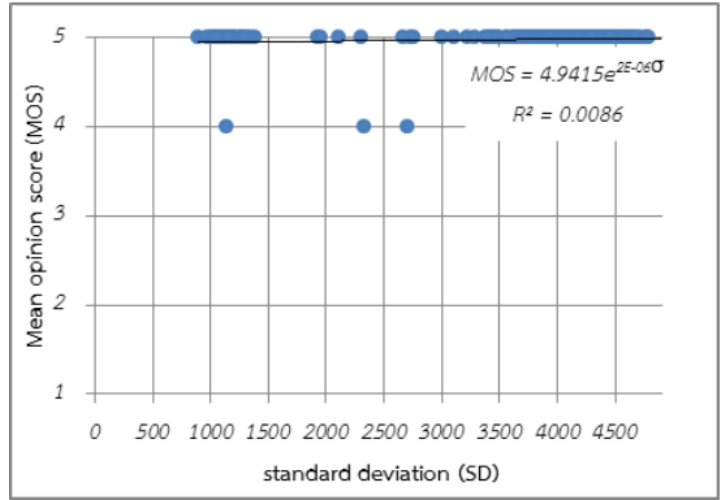

(a)

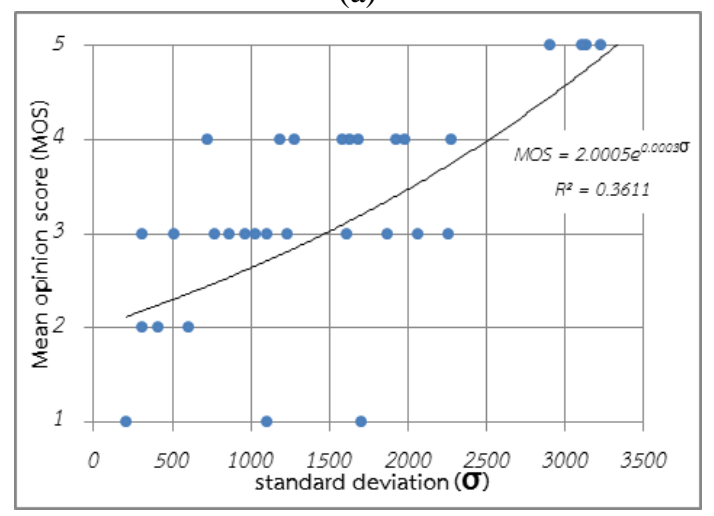

(c)

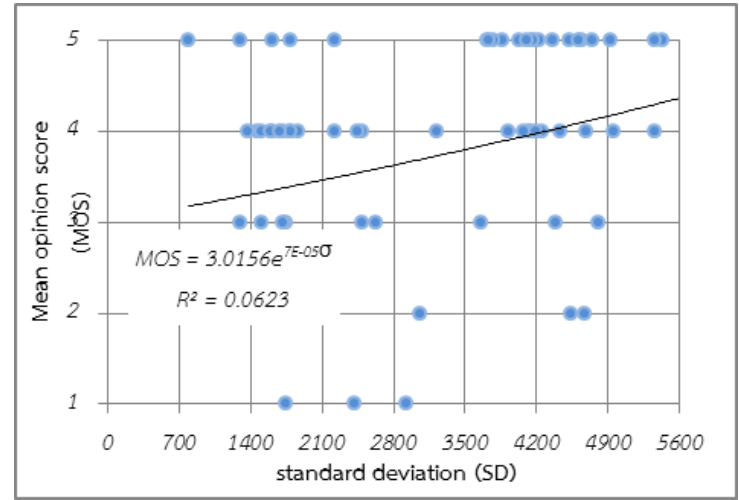

(b)

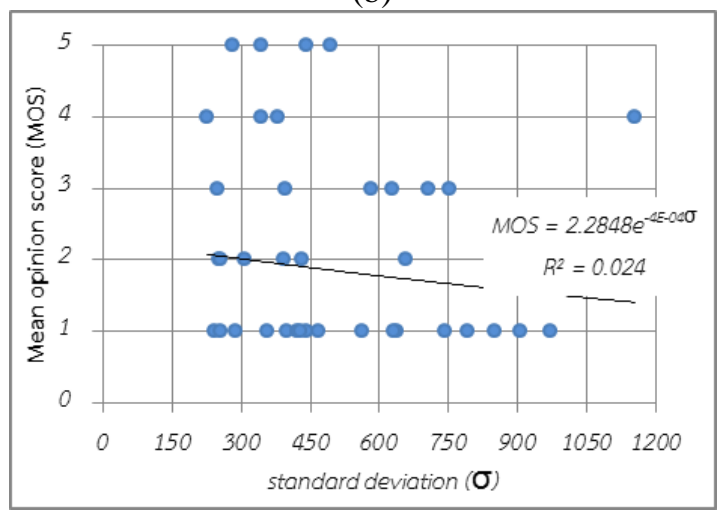

(d)

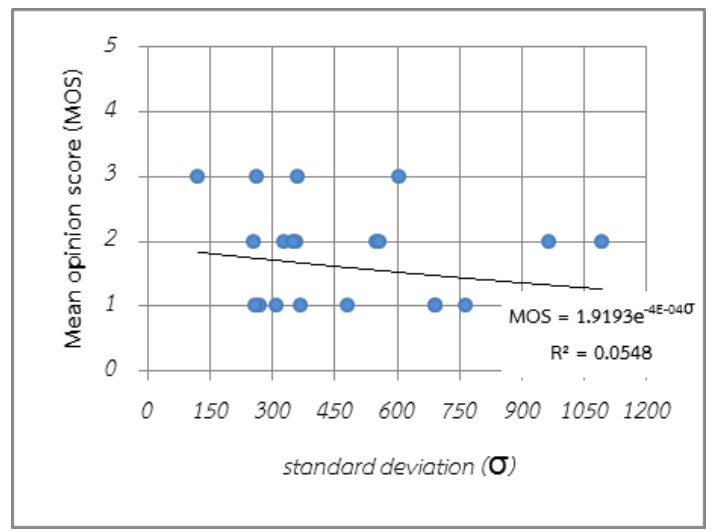

(e)

Fig. 4: the relationship between MOS and standard deviation $(\sigma)$ by subjective evaluation method for YouTube service with video quality of $1080 \mathrm{p}$, when the average throughput is a) $2100-2500 \mathrm{kbps}$ b) $1900-2100 \mathrm{kbps} \mathrm{c}$ ) $1200-1400 \mathrm{kbps}$ d) $900-1200 \mathrm{kbps}$ and e) 500-900 kbps.

For the results in Fig. 4 (b) and (c), the more standard deviation is the more MOS is achieved. The results in his are because the throughput is still high enough to play main role on the effect of fluctuation. In turn, the result in Fig. 4 (d) and (e) show that the more fluctuation is the less MOS is achieved. It is obvious that the buffer cannot help and also the average throughput is too low to notice all fluctuation effects. 


\section{Conclusion}

This paper provides the new insight on the impact of fluctuation on the user satisfaction. This work analyses QoE based on subjective evaluation method for YouTube service in 4G mobile networks. The measured results show that the fluctuation will play a main role on the user satisfaction only when the average throughput is low enough. The throughput fluctuation could not affect the satisfaction of users when the average throughput is too high. This conclusion is very helpful for service providers to neglect the unavoidable fluctuation by keeping their average throughput higher than $2100 \mathrm{kbps}$.

\section{References}

[1] "Cisco Visual Networking Index: Global Mobile Data Traffic Forecase Update 2014-2019", Cisco Inc, Feb. 2015, [online] Available: http://www.cisco.com/c/en/us/solutions/collateral/service-provider/visual-networking-indexvni/white_paper_c11-520862.html.

[2] Yan Chen, Toni Farley and Nong Ye, "QoS Requirements of Network Applications on the Internet," IOS Press and the authors, pp. 55-76, 2004.

[3] Xu, Lina, and Yantao Qiao. "Negotiator based Quality of Experience framework for video." 2016 13th IEEE Annual Consumer Communications \& Networking Conference (CCNC). IEEE, 2016.

[4] P. Casas, A. Sackl et al., "On the Quest for New KPIs in Mobile Networks: The Impact of Throughput Fluctuations on QoE”, IEEE ICC 2015

[5] A. Sackl et al., "The Influence of Network Quality Fluctuations on Web QoE”, in QoMEX, 2014.

[6] International Telecommunication Union - Telecommunication standardization Sector: Quality of Service and Dependability Vocabulary, ITU-T Rec. E. 800, November, 1988.

[7] Rec I. P. 10: Vocabulary for performance and quality of service, Amendment 2: New definitions for inclusion in Recommendation ITU-T P. 10/G. 100[J]. Int. Telecomm. Union, Geneva, 2008.

[8] ITU-T P.862, "Perceptual evaluation of speech quality (PESQ)," Feb 2001. [10] ITU-T G.107, "The E-model, a computational model for use in transmission planning," Mar 2005

[9] P. Casas, A. Sackl et al., "Negotiator based Quality of Experience Framework for Video", IEEE CCNC 2016 13th

[10] International Telecommunication Union - Telecommunication "Mean Opinion Score(MOS) terminology," ITU-T Rec. P. 800, July 2. 